\title{
Artificial life forms: the strains of the future?
}

\author{
KALYANI RAJALINGHAM \\ McGill University, Department of Medicine, Montreal, Quebec, Canada
}

\begin{abstract}
Synthetic biology is the field of biology that is involved in the manufacturing of genomic material designed by man with subsequent introduction into a chassis. A synthetic life has a minimal genome of about 500-800 genes as well as a few additional genes required to fulfill a particular task. Synthetic life forms can be used for the production of valuable target compounds such as the antimalarial drug artemisinin. This technology is applicable to all subfields of biotechnology and biology, but it is, in particular, relevant for the making of target compounds; it can be used for the making of biofuels, drugs, energy, artificial photosynthesis, in medicine, bioremediation/biosensors, in the chemical industry, and in agriculture. It is believed that these novel artificial strains will become the ruling strains in the near future with many a use in many a field. This paper outlines the positive aspects of synthetic life forms as well as the methods used to generate artificial life forms.
\end{abstract}

Key words: artificial life, synthetic life

\section{Introduction}

A newcomer to the field of biology, and still in its infancy, synthetic genomics is the field of biology that attempts to create life from non-living materials. Synthetic life possesses a man-made genetic code. After implanting a synthetic genome - fabricated genome - into an existing recipient cell, the recipient cell can then be infused with human tissue, or into another substance. Currently, the minimal set - the minimal number of genes required to sustain life - is being determined; this is carried out mainly in bacteria by waiving those genes that are not crucial for subsistence. To date, the minimal set involves about 500-800 genes; however, it can be lessened to about 300-400 genes. Desired genes are then added to the minimal set - fabricated from scratch - to elicit a particular function. It is believed that in the future, a "core genome" will be available in stock for the incorporation of desired target genes. Two approaches are used to determine the minimal set: the top-down and the bottom-up approach. In the top-down approach, one gene at a time is eliminated until the minimal set or essential genes are isolated; in the bottom-up approach, a gene at a time is assembled until a functional cell is created (Porcar et al., 2011). However, at times, eliminating two non-necessary genes can cause lethality; the degree of lethality is as such noted (Acevedo-Rocha et al., 2013). The associated problems are organization, codon bias, and conformation (Acevedo-Rocha et al., 2013). The minimal set includes the core/required genes as well as accessory genes (Juhas et al., 2012). Typically, these include 1) genes involved in DNA replication, transcription, RNA processing, aminoacyl-tRNA formation, protein folding, cell division, and cell membrane biosynthesis and 2) genes required for translation, and ribosomal synthesis, which are thought to be essential (Juhas et al., 2012).

The use of this technology in the field of biotechnology could solve many a problem. Currently, in the production of biofuels or drugs, the fact that the carbon source is used for alternate processes implies a reduction in efficiency. Therefore, scientists have been attempting to re-route the production pathways for increased production of the target compound. With the arrival of synthetic life forms, a minimal set of genes could be supplemented with the genes required for production of the target compound - that would ensure higher produc-

Corresponding author: McGill University, Department of Medicine, 1110 Pine Avenue West, Room 101, Montreal, Quebec, Canada H3A 1A3; e-mail: krajalingham@gmail.com 
tion/efficiencies. For instance, bacteria with synthetic genomes (minimal set and target genes) can be used for fuel production. Synthetic life can be used for energy production (e.g. synthetic cells that produce a particular compound, fuel or that perform artificial photosynthesis), in medicine (e.g. synthetic life that can manufacture drugs, or create new tissue), in the environment (e.g. synthetic cells that can detect and breakdown pollutants), in the chemical industry (e.g. production of chemicals in bulk), and in agriculture (EASAC, 2011). The latter involves the assembly of multiple molecules that, if functional, can lead to the production of a target molecule. For instance, modified yeast cells can be coaxed to produce anti-malarial drugs; this technology would trim the production costs by $90 \%$. In the second instance, the yeast Saccharomyces cerevisiae can be wheedled to produce the anti-cancer drug Taxol. The potential of synthetic genomics is immense. The goal of this paper is to present artificial or synthetic life forms as potential usable strains of the future - could these strains dominate in the near future?

\section{Digital genome design}

The genomes of synthetic life forms are designed on a computer by utilizing the known knowledge about existing sequences. Rewiring the genetic code digitally to favour production over proliferation is the main goal of synthetic biology (Ferry et al., 2012). Synthesis at the genomic level is not impossible, but combinatorial assembly is typically used. Cassettes of about $1080 \mathrm{bp}$ are created in such a manner as to also have an 80 bp overlap region. In other words, oligonucleotides are synthesized and attached together to create cassettes (Gibson et al., 2010). Multiple cassettes are therefore synthesized, and sequences are verified. Cassettes, at times, contain Not I restriction sites to allow for recombination via vectors. Registries of BioBricks (oligonucleotides with an overlapping region) are typically created and stored (storage of various kinds of BioBricks). Assembly is still the most difficult part of the process. Large-scale assembly begins with assembly of parts into genes, genes into pathways, and subsequent construction of an entire chromosome/genome (Ellis et al., 2011). The Registry of Standard Biological Parts contains approximately 5.000 parts (Norville et al., 2010). There are various ways of assembling the BioBricks: parallel as- sembly (simultaneous assembly), ordered assembly (in a particular order), pathway assembly (pathway assembled), and combinatorial assembly. Genomes are assembled using homologous recombination - transformation-assisted recombination (TAR); yeast cells and $B a-$ cillus can both be used as a chassis (Ellis et al., 2011). Gibson et al. (2010) transplanted synthetic M. mycoides DNA into $M$. capricolum and attained success by growing recipient cells on media. Gibson et al. (2010) state that the synthetic life form was capable of both logarithmic growth and reproduction; however, growth rates were not similar to the natural form.

\section{Target compound production}

Synthetic biology would permit the creation of a life form with the genetic content of our choice that is self-replicating and that in theory can produce a target compound. This would give scientists much freedom and increase the efficiency of production. Synthetic life forms are advantageous because 1) there is no need to purify intermediates, 2) target will not react with alternate compounds (typically, compounds undergo protection and de-protection steps to prevent them from reacting with other compounds produced by the cell; this is not a problem in synthetic life forms), 3) racemically pure compounds, and 4) secretion of target compound is possible (Keasling et al., 2012). Therefore, synthetic life forms have the potential to become the perfect strain for industrial production of target compounds. Chemical synthesis - the making of compounds in a laboratory by relying solely on chemical synthesis - of target compounds is deemed hard because of the need for a correct 3D structure. Improper 3D conformation of target compounds can be toxic to humans (Keasling et al., 2012). Microorganisms are typically utilized for the production of target compounds because they can generate the proper $3 \mathrm{D}$ conformation of the target compound. However, at times, the microorganism can produce the target compound but in minute quantities, which is insufficient to turn into a grand-scale production. Here, synthetic life forms could be beneficial. Unlike natural microorganisms that re-route carbon for multiple processes, synthetic life forms do not - they would utilize the carbon source for a single main process (assuming and ignoring the basic processes). In other words, available nutrients are utilized for multiple pur- 
poses/processes in natural microorganisms; and similarly, in a natural microorganism, production of a target compound is only one of the many other processes (which decreases yield). In artificial life forms that possess a minimal genome along with the genes required for target compound production, the nutrients would be used solely for the production of the target compound (basic processes aside). Schmidt (2009) stated that artificial life forms will improve bio-economy. This would, in theory, imply a higher yield, perhaps even sufficient for industrial scale production. For instance, artemisinin, also the precursor of the antimalarial drug artesunate, is produced in very low supply. Artemisinin, extracted from Artemisia annua and a precursor to the anti-malarial drug artesunate, is very expensive and laborious to isolate. With the arrival of synthetic technology, the metabolic pathway required for the production of artemisinin has been successfully introduced into yeast, and a synthetic life form capable of producing artemisinin has been created (Venton, 2014); the prices associated with the drug have also dropped substantially. In the modern day, large-scale production of artemisinin is both feasible and happening because of synthetic technology; current production is by the ton with an expected yield of 5060 tons per year in 2014 (Sanders). Further, synthetic life forms can be designed to withstand harsh conditions, to produce target compounds, or to detect and convert substances present in soil among many other functions (Ferry et al., 2012). Yet another application could be in the removal of carbon dioxide and production of biofuels by synthetic life forms (designed to consume carbon dioxide) (Venton, 2014). Synthetic life forms are also candidates for biofuel production. With the current need for biofuels, the "food versus fuel" debate (the use of edible material for the production of fuels), and the advent of second-generation biofuels, synthetic life forms can be tailored with those genes required to tackle the exact composition of the material. All in all, the potential of synthetic life forms is as immense as it is varied.

\section{Ethical and safety concerns}

In other words, scientists are playing God; they are aiming to create a novel synthetic self-sustaining and self-replicating organism. In addition, orthogonal biosystems - DNA alternatives (genetic code similar to DNA) - are already being considered. However, one cannot predict the level of risk associated with fabricated genomes and artificial cells (Cho and Relman, 2010); scientists have not yet determined the repercussions of releasing self-replicating artificial life forms in the environment, or the damage that might ensue. There are three major concerns: 1) escape from quarantined facility and the subsequent proliferation and damage to ecosystem, 2) detrimental side-effects to humans, and 3) bioterrorism (Tucker and Zilinskas, 2006). In the first case, given that a synthetic life form would constitute a genetically modified organism, there is much fear about accidental release of the novel self-replicating life form and the potential damage to ecosystems. In this case, three possibilities exist: 1) disruption of local biota, 2) invasiveness, and 3) disruption/altering natural balance of ecosystem (Tucker and Zilinskas, 2006). Precautionary measures to address this problem include the incorporation of genes that can trigger self-destruction, or the creation of life forms that require a particular and limited nutrient for growth or nutrients that are not naturally found in nature. Yet another method of control is designing the synthetic life form to use unnatural amino acids which would prevent expression outside the quarantined facility. Furthermore, Tucker and Zilinskas (2006) claim that natural microorganisms typically outcompete genetically engineered microorganisms such that even if synthetic life forms are released, they might not be able to outcompete natural residents. Yet another concern is the possibility of mutations. Mutations occurring in critical and necessary regions would very possibly result in cellular death. However, mutations in the adjunct genes could result in the production of non-target compound or no compounds at all; such issues have to be addressed.

Furthermore, the forces (e.g. CIA) fear that synthetic genomics can be utilized to engineer biological weapons (EASAC, 2011). There has been concern about virulence, about the unpredictability of genetic interactions, and about the behavior of novel creations (Cho and Relman, 2010). Another concern is bioterrorism - the production of compounds that could mar human lives. For instance, Eckard Wimmer wove together the genome of poliovirus from oligonucleotides and placed it in a viral chassis using the genetic information available, and oligonucleotides obtained from suppliers (Tucker and Zilinskas, 2006). The United States Centers for Disease Control and Prevention synthesized the genome 
of the Spanish influenza virus - a virus that killed almost 100 million people in the past. In other words, any virus or bacterium with a known genetic code can be reconstructed using the synthetic technology (Tucker and $\mathrm{Zi}$ linskas, 2006). However, the fact of the matter is that synthetic technology is already available - the knowledge that one can build a synthetic genome is already available - and as such, ignoring or preventing the use of this technology for the betterment of the world would be senseless. Because synthetic life forms can be generated by assembling oligonucleotides, the process is not one that is impossibly difficult, and the mere knowledge of the process is where the danger lies, not the technology itself. Perhaps control of genetic information or code available and use of unnatural amino acids could solve the problem, but overlooking or rejecting synthetic technology is not the solution.

Ethical debate about security risks associated with synthetic life still remains as the people wonder when they should fear the technology. Genetically modified organisms with a single transgene have yet to gain approval from both the scientific community and the population as a whole, and as such, synthetic life with a man-made genetically borrowed code from various organisms faces a similar obstacle. Another concern is that of playing God. It so seems that many are opposed to the idea of creations by humans as they believe that it is the responsibility of the almighty God to create, not that of a human. From stem cell research to cloning, opposition from the non-scientists seems to be the norm; synthetic life forms might face the same opposition. Furthermore, it can be stated that the technology that begins with microorganisms in the $21^{\text {st }}$ century could one day be used to create synthetic animals or even humans (given that synthetic life began with the sequencing project). However, if, in fact, we were to abide by the fears of the society, then genetically modified organisms and synthetic life forms must be banned altogether along with their uses. Perhaps the implementation of cautionary measures would be a better idea than banning the technology.

\section{Conclusions}

Synthetic life forms have the potential to generate target compounds that have, to date, been difficult to obtain such as artemisinin. In fact, in the modern day and age, synthetic life forms are being generated and their potential uses are being determined. Given the vast potential of synthetic life forms, it would be rather senseless to ignore the technology out of fear. I believe that this technology has much potential, and that with control (rules/regulations), it could benefit the society.

\section{Acknowledgments}

The author wishes to thank her husband Dr. William Kane and her mother Rajaluxmy Rajalingham for reviewing this paper.

\section{References}

Acevedo-Rocha C., Fang G., Schmidt M., Ussery D., Danchin A. (2013) From essential to persistent genes: a functional approach to constructing synthetic life. Trends Genet. 29: 273-279.

Cho M., Relman D. (2010) Synthetic "life", ethics, National Security, and public discourse. Science 329.

EASAC (2011) Synthetic Biology: An introduction, European Academies Science Advisory Council.

Ellis T., Adie T., Baldwin G. (2011) DNA assembly for synthetic biology: from parts to pathways and beyond. Integrat. Biol. 3: 109.

Ferry M., Hasty J., Cookson N. (2012) Synthetic biology approaches to biofuel production. Biofuels 3: 9-12.

Gibson D. et al. (2010) Creation of a bacterial cell controlled by a chemically synthesized genome. Science 329: 52-56.

Juhas M., Eberl L., Church G. (2012) Essential genes as antimicrobial targets and cornerstones of synthetic biology. Trends Biotechnol. 30: 601-607.

Keasling J., Mendoza A., Baran P. (2012) Synthesis: A constructive debate. Nature 492: 188-189.

Porcar M., Danchin A., de Lorenzo V., dos Santos V., Krasnogor N., Rasmussen S., Moya A. (2011) The ten grand challenges of synthetic life. Syst. Synth. Biol. 5: 1-9.

Norville J., Derda R., Gupta S., Drinkwater K., Belcher A., Leschziner A., Knight T. (2010) Introduction of customized inserts for streamlined assembly and optimization of BioBrick synthetic genetic circuits. J. Biol. Engin. 4: 17.

Sanders R. (2013) Launch of antimalarial drug a triumph for UC Berkeley, synthetic biology. Berkeley News: 21 April.

Schmidt M. (2009) Special issue: societal aspects of synthetic biology. Syst. Synth. Biol. 3: 1-2.

Tucker J., Zilinskas R. (2006) The promise and perils of synthetic biology. The New Atlantis, 25-45.

Venton D. (2014) Core Concept: Synthetic biology - change, accelerated. Proc. Nat. Acad. Sci. 111: 16978-16979. 